\title{
Q-switched erbium doped fiber laser based on single and multiple walled carbon nanotubes embedded in polyethylene oxide film as saturable absorber
}

\author{
M.H.M. Ahmed ${ }^{a}$, N.M. Ali ${ }^{a}$, Z.S. Salleh ${ }^{\text {a }}$, A.A. Rahman ${ }^{\text {b }}$, S.W. Harun ${ }^{a}$, M. Manaf ${ }^{b}$, H. Arof $^{\text {a,* }}$ \\ a Department of Electrical Engineering, Faculty of Engineering, University of Malaya, 50603 Kuala Lumpur, Malaysia

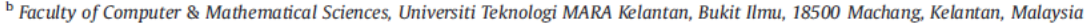

A R T I C L E I N F O

Article history:

Received 20 April 2014

Received in revised form

30 May 2014

Accepted 1 July 2014

Available online 19 July 2014

Keywords:

Single-walled and multi-walled carbon

nanotubes

Q-switching

Passive saturable absorber

\begin{abstract}
A B S T R A C T
A passive, stable and low cost Q-switched Erbium-doped fiber laser (EDFL) is demonstrated using both single-walled carbon nanotubes (SWCNTs) and multi-walled carbon nanotubes (MWCNTs), which are embedded in polyethylene oxide (PEO) film as a saturable absorber (SA). The film is sandwiched between two FC/PC fiber connectors and integrated into the laser cavity for Q-switching pulse generation operating at wavelength of $1533.6 \mathrm{~nm}$. With SWCNTs, the laser produces a stable pulse train with repetition rate and pulse width ranging from 9.52 to $33.33 \mathrm{kHz}$ and 16.8 to $8.0 \mu$ s while varying the $980 \mathrm{~nm}$ pump power from $48.5 \mathrm{~mW}$ to $100.4 \mathrm{~mW}$. On the other hand, with MWCNTs, the repetition rate and pulse width can be tuned in a wider range of $6.12-33.62 \mathrm{kHz}$ and $9.5-4.2 \mu \mathrm{s}$, respectively as the pump power increases from 37.9 to $120.6 \mathrm{~mW}$. The MWCNTs produce the pulse train at a lower threshold and attain a higher repetition rate compared to the SWCNTs. This is due to thicker carbon nanotubes layer of the MWCNTs which provides more absorption and consequently higher damage threshold. The Q-switched EDFL produces the highest pulse energy of $531 \mathrm{~nJ}$ at pump power of $37.9 \mathrm{~mW}$ with the use of MWCNTs-PEO SA.
\end{abstract}

c 2014 Elsevier Ltd. All rights reserved.

\section{Introduction}

Q-switched EDFLs have gained a tremendous research interest in recent years for their potential applications in metrology, fiber sensor, optical communication, medical diagnostics and others [1-4]. Passively Q-switched EDFLs have attracted much attention for their advantages of compactness, low cost, flexibility and simple design over their actively Q-switched counterparts $[4,5]$. Different kinds of saturable absorbers (SAs), such as the transition metal-doped crystals [1] and semiconductor quantum-well structures [2], have been used to realize Q-switched pulses. However, these SAs require additional alignment devices, such as lens, mirrors or U-bench units when used in a laser cavity. These devices may introduce extra insertion loss and added complexity. Recently, the use of single-walled carbon nanotubes (SWCNTs) and multi-walled carbon nanotubes (MWCNTs) material as $\mathrm{SAs}$ has captured the interest of many researchers working in both $\mathrm{O}$ switched and mode-locked fiber lasers [6-8]. This is due to a number of inherent advantages that they possess, including good compatibility

\footnotetext{
${ }^{*}$ Corresponding author. Tel.: +60379674456 .

E-mail address: ahamzah@um.edu.my (H. Arof).

http://dx.doi.org/10.1016/j.optlastec.2014.07.00

0030-3992/○ 2014 Elsevier Ltd. All rights reserved.
}

with optical fibers, low saturation intensity, fast recovery time, and wide operating bandwidth.

Compared to SWCNTs, the MWCNTs have higher mechanical strength, better thermal stability and are able to absorb more photons per nanotube due to the higher mass density of the multiwalls. MWCNT material also has good thermal characteristics, which are important for high power ultrafast laser development. These favorable features are due to the structure of MWCNTs which takes the form of a stack of concentrically rolled graphene sheets. The outer walls protect the inner walls from damage or oxidation so that the thermal or laser damage threshold of MWCNT is higher than that of the SWCNTs $[9,10]$. Furthermore, the preparation of the MWCNT material does not involve complicated techniques or special growing conditions such that its production yield is high for each growth. Consequently, the production cost of MWCNT material is about $50-20 \%$ of that of SWCNT material [11].

To date, there are only a few reported works on using MWCNTs material to construct a saturable absorber. For instance, Lin et. al. [12] employed multi-walled MWCNTs based saturable absorber (SA) for mode locking of a $\mathrm{Nd}: \mathrm{YVO}_{4}$ laser. Very recently, diode pumped passively Q-switched Nd:LuAG lasers with MWCNTs as SA were also demonstrated [13,14]. For instance, Chen et. al. 
demonstrated Q-switched laser operating at $1064.1 \mathrm{~nm}$ with a pulse as short as $78 \mathrm{~ns}$ and a repetition rate of about $100 \mathrm{kHz}$ [14] In this paper, two different Q-switched erbium-doped fiber lasers (EDFLs) are demonstrated using both SWCNTs and MWCNTs-based SAs, which are embedded in polyethylene oxide (PEO) composite. The $\mathrm{SA}$ is constructed by sandwiching the composite film between two fiber connectors. Compared to previous works, the proposed laser operates at $1550 \mathrm{~nm}$ region which is desirable for communication applications by using all-fiber configuration.

\section{Experimental setup}

Fig. 1 shows the schematic configuration of the Q-switched EDFL, which uses either SWCNT or MWCNT SAs as the Q-switcher. A $1 \mathrm{~m}$ long highly doped Erbium-doped fiber (EDF) with absorption coefficients of 23 and $35 \mathrm{~dB} / \mathrm{m}$ at 980 and $1531 \mathrm{~nm}$ wavelength, is used as the gain medium. It is pumped by a commercial $980 \mathrm{~nm}$ laser diode with a maximum pump power of $125 \mathrm{~mW}$ via a 980/1550 wavelength division multiplexer (WDM). An optical isolator is used to avoid the backward reflection and ensure unidirectional operation. The laser light is extracted from the cavity by a $95 / 5$ fiber coupler which retains only $5 \%$ of the light in the cavity for further oscillation. An optical spectrum analyzer (OSA) with wavelength resolution of $0.05 \mathrm{~nm}$ is used to capture the output laser spectrum while a $350 \mathrm{MHz}$ oscilloscope in conjunction with $1.2 \mathrm{GHz}$ bandwidth photo-detector is used to detect the pulse train. The total length of the cavity is around $12 \mathrm{~m}$. In order to avoid passive mode-locking, it is worth noting that we intentionally use a $95 / 5$ coupler, which allow only $5 \%$ of the light to oscillate in the cavity. This induces a cavity loss of more than $15 \mathrm{~dB}$ in the laser cavity, which is sufficient enough to avoid modelocking.

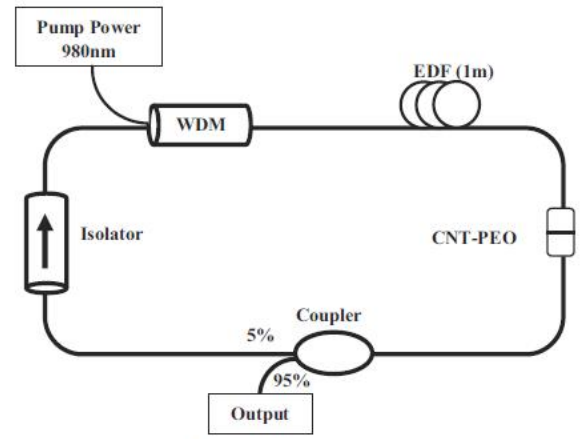

Fig. 1. Experimental setup of the Q-switched EDFL configured with either SWCNTs or MWCNTs based SA as the Q-switcher.
The main component of the proposed EDFL system is the SA which is obtained by embedding either SWCNTs or MWCNTs into PEO polymer film. To match the EDFL operating at $1550 \mathrm{~nm}$, choosing suitable carbon nanotubes with correct mean diameter, length and distributed diameter range is a critical step. In this work, we used SWCNTs with a distributed diameter of 1-2 nm and length of 3-30 $\mu \mathrm{m}$ while the MWCNTs used have a diameter of $10-$ $20 \mathrm{~nm}$ and length of $1-2 \mu \mathrm{m}$. The host material was polyethylene oxide (PEO), which has excellent film forming, emulsifying, and adhesive properties. The carbon nanotubes material used for the fabrication of the SA in this experiment is functionalized so that it can be dissolved in water. The functionalize solution was prepared by dissolving $4 \mathrm{~g}$ of sodium dodecyl sulfate (SDS) in $400 \mathrm{~m}$ deionized water. Fabricating the SA involved three major steps. Firstly, we mix the CNTs and dispersant in water to form a hybrid solution. We stirred the hybrid solution by using ultrasonic cleaner for $1 \mathrm{~h}$ to uniformly disperse CNTs with suitable ratio of al inclusions. The solution was centrifuged at $1000 \mathrm{rpm}$ to remove large particles of undispersed CNTs to obtain dispersed suspension that is stable for weeks. Secondly, we prepared a PEO solution by dissolving $1 \mathrm{~g}$ of PEO with an average molecular weight of $1 \times 10^{6} \mathrm{~g} / \mathrm{mol}$ in $120 \mathrm{ml}$ distilled water. Then we mixed the PEO solution with the hybrid carbon nanotubes solution to form the precursor and the mixture was stirred using ultrasonic cleaner for about $1 \mathrm{~h}$. This step helped us get precursor with enough viscosity so that it could be easily used in forming the carbon nanotube polymer film. Finally, suitable amounts of precursor were spread thinly on the glass substrate, and let dry in the room temperature to form the SA film.

\section{Results and discussion}

Fig. 2(a) and (b) shows the Field Emission Scanning Electron Microscopy (FESEM) images of the SWCNTs and MWCNTs film, respectively. Both figures indicate that the carbon nanotubes are randomly distributed in the polymer composite. The SA was fabricated by cutting a small part of the prepared composite film $\left(2 \times 2 \mathrm{~mm}^{2}\right)$ and sandwiching it between two $\mathrm{FC} / \mathrm{PC}$ fiber connectors, after depositing index-matching gel onto the fiber ends.

Raman spectroscopy was then performed on both films using laser excitation at $532 \mathrm{~nm}$ to confirm the presence of carbon nanotubes in the composite film. Fig. 3 compares the Raman spectrum of the SWCNTs film with the MWCNTs film. The spectrum for the SWCNTs film shows the distinct feature of the G peak, which originates from the tangential vibrations of the carbon atoms, at $1586 \mathrm{~cm}^{-1}$. In the low frequency region, the RBMs are observed, for which their energy is inversely related to SWCNT diameter. Since the distinct peaks are observed at around $214 \mathrm{~cm}^{-1}$, the SWCNT diameter is estimated to be approximately $0.9 \mathrm{~nm}$. This translates to the operating wavelength of around $1550 \mathrm{~nm}$ region. The carbon and $G^{\prime}$ peaks are also observed at $1363 \mathrm{~cm}^{-1}$ and
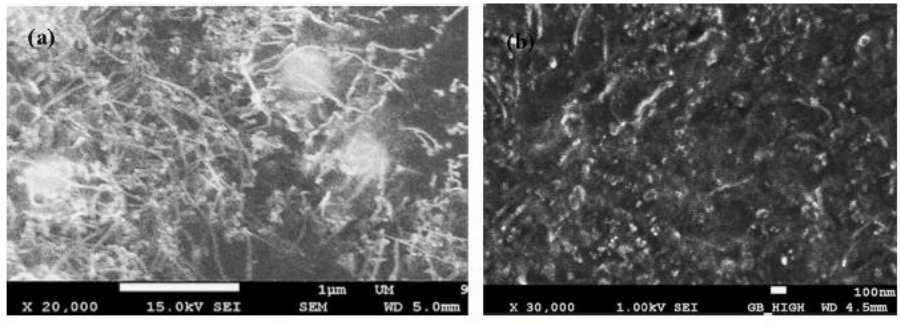

Fig. 2. FESEM images of (a) the SWCNTs-PEO and (b) MWCNTs-PEO composite film, 


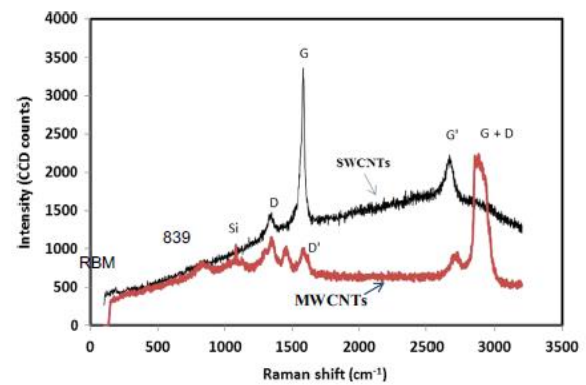

Fig. 3. Raman spectroscopy spectrum for both SWCNTs-PEO and MWCNTs-PEO composite film.

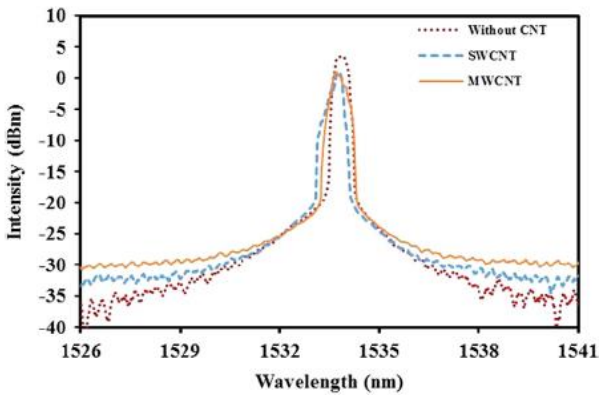

Fig. 4. Optical spectra of the Q-switched EDFL configured with SWCNT and MWCNT SAs as well as without the SA at pump power $100.4 \mathrm{~mW}$.

$2670 \mathrm{~cm}^{-1}$, respectively. On the other hand, the Raman spectrum of the MWCNTs film bears a lot of resemblance to that of graphene, which is not surprising as the MWCNT has many layers of graphene wrapped around the core tube. We can see well defined G $\left(1586 \mathrm{~cm}^{-1}\right)$ and $\mathrm{G}^{\prime}\left(2705 \mathrm{~cm}^{-1}\right)$ bands in the figure as normally associated with graphene and graphite. We also see a prominent band around $1350 \mathrm{~cm}^{-1}$, which is known as the D band. This indicates that the carbon nanotubes type is multi-walled, with multi-layer configuration and disordered structure. As expected, the radial breathing mode (RBM) bands, which correspond to the expansion and contraction of the tubes, are not present in the MWCNT because the outer tubes restrict the breathing mode. The $\mathrm{D}^{\prime}$ band which is a weak shoulder of the G-band is also observed at $1608 \mathrm{~cm}^{-1}$ due to double resonance feature induced by disorder and defect. In addition, other distinguishable features like $D+G$ band $\left(2880 \mathrm{~cm}^{-1}\right)$, a small peak at $839 \mathrm{~cm}^{-1}$ and Si were also observed as depicted in Fig. 3.

At first, the performance of the ring EDFL is investigated by varying the $980 \mathrm{~nm}$ pump power. Initially, the continuous wave (CW) lasing was observed at pump power threshold of $26 \mathrm{~mW}$. A stable and self-starting Q-switching operation is then obtained just by adjusting the pump power over thresholds of $48.5 \mathrm{~mW}$ and $37.9 \mathrm{~mW}$ for the SWCNTs and MWCNTs SA, respectively. Fig. 4 shows the output spectra of both Q-switched EDFLs configured with SWCNTs and MWCNTs SA when the pump power is fixed at $100.4 \mathrm{~mW}$. The output spectrum of the CW EDFL, which is obtained by removing the SA is also included for comparison purpose. As shown in Fig. 4, both Q-switched EDFLs operate at $1533.6 \mathrm{~nm}$, which is slightly shifted to a shorter wavelength compared to the CW EDFL. This is attributed to the presence of the SA, which increases the cavity loss and thus adjusts the lasing wavelength to a shorter value, with a slightly higher gain to

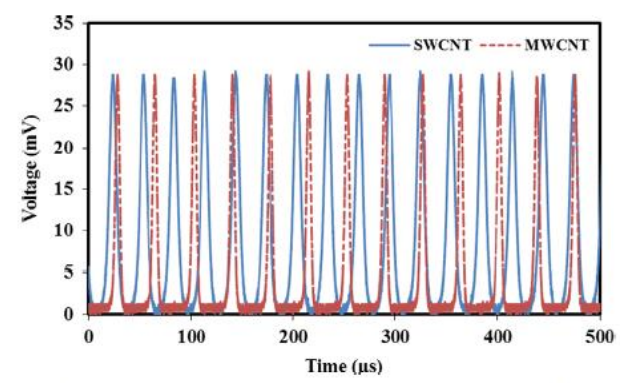

Fig. 5. Pulse trains for SWCNT and MWCNT at pump power $100.4 \mathrm{~mW}$.

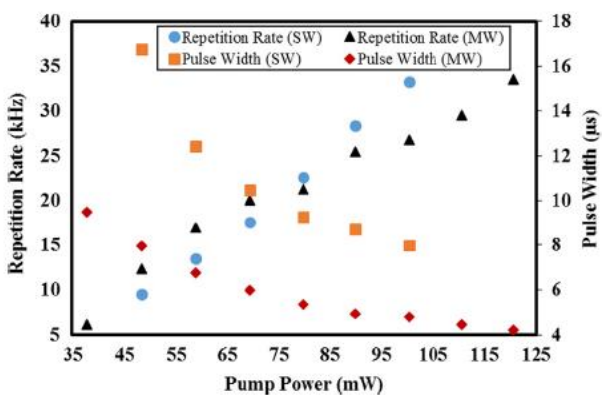

Fig. 6. Repetition rate and pulse width as functions of pump power for SWCNTsand MWCNTs-based EDFLs.

compensate for the loss. The $3 \mathrm{~dB}$ bandwidths of the output spectra are $0.47 \mathrm{~nm}, 0.68 \mathrm{~nm}$ and $0.70 \mathrm{~nm}$ for the EDFL configured without CNT, with SWCNTs and with MWCNTs respectively. Spectral broadening is also observed in both Q-switched EDFLs due to the self-phase modulation effect in the cavity. Compared to the CW operation without the SA, the peak power of both Qswitched EDFLs is also degraded due to the increase in the total loss in the ring cavity. It is also found that, both Q-switched EDFLs operate almost at the same peak wavelength and output peak power. This indicates that both SWCNTs and MWCNTs SA have a similar insertion loss.

Fig. 5 compares the oscilloscope traces of Q-switched pulse trains obtained by the SWCNTs and MWCNTs SA at pump power of $100.4 \mathrm{~mW}$. It is observed that the Q-switching operation is stable for both EDFLs without any distinct amplitude modulation in each Q-switched envelop of the spectrum. This indicates that the selfmode locking effect on the Q-switching is suppressed. The durations between two pulses are measured to be around $30.0 \mu \mathrm{s}$ and $37.3 \mu \mathrm{s}$, which can be translated to repetition rates of $33.33 \mathrm{kHz}$ and $26.82 \mathrm{kHz}$, for the EDFL with SWCNTs and MWCNTs SA respectively. Fig. 6 shows how repetition rate and pulse width are related to the pump power for both Q-switched lasers. The pulse repetition rate is seen to increase almost linearly with the pump power, while the pulse width decreases almost linearly with the pump power. This agrees well with the theory of passive Q-switching with saturable absorber. The pulse repetition rate of the SWCNTs-based Q-switched EDFL can be widely tuned from $9.52 \mathrm{kHz}$ to $33.33 \mathrm{kHz}$ by varying the pump power from $48.5 \mathrm{~mW}$ to $100.4 \mathrm{~mW}$. On the other hand, the pulse width reduces from 16.77 to $8.0 \mu \mathrm{s}$ as the pump power increases from 48.5 to $100.4 \mathrm{~mW}$. The Q-switching pulse disappears as the pump power is increased above $100.4 \mathrm{~mW}$.

Link to Full-Text Articles:

http://www.sciencedirect.com/science/article/pii/S0030399214001704

http://adsabs.harvard.edu/abs/20150ptLT..65...25A 